\title{
Is the grass greener on the other side?
}

\author{
Encouraging the development of synthetic biology in Europe
}

$\mathrm{E}$ arlier this year, researchers at the J. Craig Venter Institute (Rockville, MD, USA) published the synthesis of an artificial genome for Mycoplasma genitalium, the smallest free-living bacterium (Gibson et al, 2008). They did not create artificial life, but they did take an important step towards that elusive goal, the most high-profile and controversial application of synthetic biology. More prosaically, but importantly, synthetic biology also offers the prospect of engineering organisms able to turn refuse into biofuels; scrubbing excess carbon from the atmosphere; creating new drugs, artificial organs and other cell-based therapeutics; and developing highly efficient biosynthetic catalysts.

Not surprisingly, the enormous commercial potential of synthetic biology is beginning to create considerable excitement, reminiscent of the advent of genetic engineering in the 1980s and 1990s. At first sight, it might seem that most of the research and commercialization associated with this new field takes place in the USA. The Biobricks Foundation (Cambridge, MA, USA) - an initiative to standardize the DNA 'building blocks' that encode basic biological functions for engineering purposes, and that attracted considerable media and scientific attention-was founded by engineers and scientists from the Massachusetts Institute of Technology (MIT; Cambridge, MA, USA), Harvard University (Cambridge, MA, USA) and the University of California (San Francisco, CA, USA). The USA also contains a considerable number of laboratories and researchers investigating synthetic biology (www.syntheticbiology.org). US scientists and venture capitalists have founded several companies to exploit synthetic biology's commercial potential—such as Synthetic Genomics (La Jolla, CA, USA), Codon Devices (Cambridge, MA, USA) and Amyris (Emeryville, CA, USA).

Although it might seem that the USA is ahead in the race to reap the possible benefits of this emerging field, Europe is not necessarily about to lose out. However, the future of synthetic biology in Europe depends as much on regulatory structures, public acceptance, funding and research networking as it does on scientific advances and commercial development. In response, various institutions, including the European Union (EU), are beginning to create the necessary scientific and regulatory infrastructure to support synthetic biology.

S ynthetic biology has its roots in classical genetic engineering; systems biology and functional genomics provide the data and architectural rules that allow bioengineers to design artificial biological constructs. Indeed, synthetic and systems biology show numerous synergies: both focus on systems, rather than on individual genes or proteins; both conceptualize cellular processes as circuits and networks; and both use modelling to analyse functions and functional systems (O'Malley et al, 2008). Yet, there are also clear differences. "Systems biology embraces complexity, synthetic biology often attempts to do the opposite," commented Maureen O'Malley, a research fellow at the Economic and Social Research Council Centre for Genomics and Society (Egenis) at the University of Exeter in the UK. "Synthetic biology is, in many respects, the other side of the coin of systems biology."

Synthetic biology also shows conceptual similarities with electronic engineering. "Over the last five years or so, researchers started working on standardized artificial genetic elements to construct relatively simple and reliable artificial logic devices and systems," c o m m e n t ed Hubert Bernauer, Chief Executive

Officer of ATG:biosynthetics, a biotechnology company in Merzhausen, Germany. "The concept is similar to IT engineers using offthe-shelf transistors and microprocessors."

The potential impact of synthetic biology could be as profound as the computer revolution. Some researchers, for example, plan

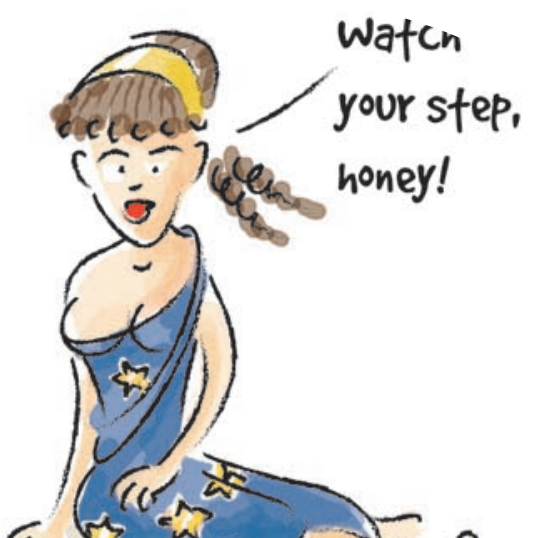


artificial biological sensors that can measure levels of certain molecules and respond by producing or releasing other compounds to maintain optimal levels (Benenson et al, 2004), which could be developed into sophisticated drug delivery systems. "Artificial biological sensor-actuator systems hosted in stem-cells could measure glucose and release a precise dose of insulin. This would avoid the need for invasive injections and maintain an adaptive physiological response," Bernauer said. "Biologists could also use standardized artificial genetic elements to design cellular systems that attack tumour cells, or remove the plaques and neurofibrillatory tangles that cause Alzheimer's disease."

\section{...the enormous commercial potential of synthetic biology is beginning to create considerable excitement, reminiscent of the advent of genetic engineering in the 1980s and 1990s}

Synthetic biology can also facilitate the synthesis of complex therapeutic molecules such as the anti-malaria drug artemisinin, which is too expensive for many malaria patients in impoverished countries. A research team led by Jay Keasling at the University of California, Berkeley, USA, has modified the metabolic pathways of Escherichia coli to produce a precursor of artemisinin. A publicprivate consortium is now developing the technology into a commercial application to produce low-cost, semi-synthetic artemisinin through fermentation (Hale et al, 2007).

Synthetic biology might even allow researchers to go beyond the existing forms of life. "Synthetic biology may provide a way of exploring the spaces of biological possibility that evolution has not explored in the history of life on earth," said Dan Nicholson, a PhD student at Egenis. "Synthetic biologists would then need to distinguish the possibilities that have not been realised by evolutionary processes for contingent or historical reasons from those which evolution did not realise because of the constraints imposed by physical and biological laws."

However, "most applications of synthetic biology suggested so far are perhaps futuristic," warned Paul Freemont, Head of the Division of Molecular Biosciences and a member of the advisory board of the Institute of Systems and Synthetic
Biology at Imperial College London, UK. "Nevertheless, there's no doubt that some of the suggested applications are potentially critical for the future survival of the planet. Synthetic biology could offer a means to capture atmospheric carbon, degrade pollutants or to improve the yield of food in environmentally challenging areas."

T he considerable commercial and scientific potential of synthetic biology promoted a spate of European initiatives to stimulate further research and commercialization. "Europe is developing a strategy for synthetic biology that should be ready for the next funding framework," Freemont commented. "In the meantime, European efforts in systems biology are beginning to bear fruit. Synthetic biology will benefit from this success: there are clear synergies between the two."

Nevertheless, not all the elements that underpin efficient research are yet in place. The design and construction of artificial devices requires detailed knowledge of biological systems (O'Malley et al, 2008); as a result, synthetic biology will use and generate vast amounts of data. "Successful systems and synthetic biology needs to integrate many different data types," said Janet Thornton, Director of the European Molecular Biology Laboratory's European Bioinformatics Institute at Hinxton in the UK. "The need for an enhanced infrastructure to capture, store, distribute and then exploit biological data in Europe is overwhelming. Successfully integrating the various disparate data will ultimately provide the route for understanding the molecular basis of life and for engineering artificial constructs."

\section{The considerable commercial and scientific potential of synthetic biology promoted a spate of European initiatives to stimulate further research and commercialization}

Indeed, the European Strategic Forum for Research Infrastructures (ESFRI)—a group of scientists convened to inform EU science policy-making -identified 'biological information provision' as a priority for Europe. In response, the EU began a preparatory project called ELIXIR (http://www.elixir-europe.org) to construct and operate a sustainable infrastructure for biological information across
Europe. ELIXIR includes, for example, distributed and centralized databases and networks, and supercomputers, and aims to develop further and exploit the data grid and the semantic web-a version of the internet that computers can use to find, share and combine information without human direction and intervention. "If successful in raising funds, ELIXIR will support life science research and its translation to medicine and the environment, bio-industries and society," said Thornton, who is the coordinator of ELIXIR. "As the amount of data grows, the need for massive databases and associated processing hardware will increase. We need to 'bite the bullet' in Europe and provide the data infrastructure to adequately support this endeavour."

S everal other initiatives also aim to ensure that the EU remains in the vanguard of synthetic biology-for example, by overcoming the fragmented nature of research in synthetic biology, which involves groups in different countries working in various disciplines. The Biotechnology and Biological Sciences Research Council in the UK (BBSRC; Swindon, UK) and, at a European level, the Towards a European Strategy for Synthetic Biology initiative (TESSY; Karlsruhe, Germany) are creating multidisciplinary networks and raising awareness. Similarly, EMERGENCE (http://www.emergence.ethz.ch/) provides a "communication and working platform" that considers education, infrastructure and standardization to strengthen the organization and concepts that underpin synthetic biology. Meanwhile, SYNBIOLOGY (http:// www2.spi.pt/synbiology/) examines the commercial prospects for synthetic biology and aims to identify factors that would increase effective research.

"Bringing together academic groups working in synthetic biology, for example using the EU Framework system, would be useful," commented Jamie Davies, Professor of Experimental Anatomy at the University of Edinburgh in Scotland. Yet, Davies also warned against too much bureaucratization and organization. "In a fast moving field, the prevailing rather bureaucratic culture of milestones and deliverables can seem horribly inflexible," he said. "The field is so young I think it would be better to support it in as bottomup a way as possible. Setting top-down strategies may stifle rather than encourage development." 
Any technology as potentially powerful as synthetic biology also raises the threat of deliberate abuse or accidental damage to human health or the environment. In response, researchers in the USA and Europe have been examining ways to ensure biosecurity and biosafety. SYNBIOSAFE (http://www.synbiosafe.eu/) examines and encourages debate around ethics, public awareness, safety and security. Similarly, the Industry Association of Synthetic Biology (IASB; http://www.iv-synbio.org/), founded by six European companies, is also considering ethical and safety issues; it held a workshop in April 2008 to discuss biosecurity issues that arise from the use of synthetic genes.

$\mathrm{T}$ hese initiatives depend on an efficient and flexible regulatory framework. "Europe urgently needs to establish a robust governance framework to maintain public confidence," said Paul Martin, Deputy Director of the Institute for Science and Society at the University of Nottingham in the UK, and co-author of a recent report on the ethical and social challenges of synthetic biology (Balmer \& Martin, 2008). Bernauer pointed out that various surveys, including those by TESSY and SYNBIOLOGY, suggest that most researchers in synthetic biology favour a unified European approach to regulation, rather than country-specific initiatives. However, he emphasized that European regulators need to avoid an overly restrictive framework that stifles research. "In my opinion, there are no real dangers if there are clear rules about how the technology has to be handled," Bernauer remarked. "In green biotech, there is no documented threat from the technology itself. The problems arose from misconduct on the market."

\section{Europe urgently needs to establish a robust governance framework to maintain public confidence...}

Synthetic biologists claim that they are cognizant of the potential hazards and engineer safeguards into their artificial biological systems. For example, the artificial $M$. genitalium genome created at the Venter Institute-also called M. laboratorium-contains all the genes of the wild-type organism, except MG408, which researchers disrupted to inhibit pathogenicity. In addition, regulatory frameworks might insist that any synthetic systems are minimal cells and orthogonalic-modifying a component should not create side effects in other components. "Orthogonality of function and minimal cell system are prerequisites for increasing safety of synthetic biology," Bernauer commented.

Although the regulations need to encompass details that are specific to synthetic biology, much of the framework might already be in place. "Genetically modified organisms [GMOs] are already well-regulated at a European level," Freemont noted. "It seems obvious that synthetic biology is an extension of this. However, we need a wide reaching public debate and an open and transparent framework. But it seems to me that we have a regulatory framework in place that we can build on and extend where necessary."

Conversely, Martin counsels against over-reliance on orthogonality and engineered safeguards to decrease the potential risks of GMOs. "Based on the 30 years of knowledge we have acquired about genetically modified bacteria the risk may be very low," he said. "However, European governance frameworks need to exercise a high level of caution in the early development of the technology. This will be crucial to maintain public support. Even one significant problem could put the field back years." Overall, the European framework should maintain flexibility, argued John Dupré, Professor of Philosophy of Science at Exeter University in the UK and Director of Egenis. "Regulation does not require absolute boundaries: it works on the basis of successful regulatory and scientific negotiation of boundaries," he said. "Governance for synthetic biology needs to be able to respond quickly and flexibly to rapidly evolving new technologies."

I n parallel with the development of a regulatory framework, synthetic biologists also need to communicate the risks and benefits clearly to the European public. "Researchers won't pull the European public along unless there are clear benefits from synthetic biology and people feel reassured that the risks have been dealt with in an open and honest fashion," Martin commented. "The main lesson from the genetically modified crop debacle is that where there are few immediate public benefits and where there appears to be powerful sectional interests at work, the public can be very reluctant to accept expert assurances that a new biotechnology is either safe or desirable." In addition, European scientists have learned another valuable lesson from the GM crop debate. "It is possible that synthetic biology may not always deliver-so we need to be careful not to unreasonably raise the expectations of the public and the funders," Freemont said.

\section{...European regulators need to avoid an overly restrictive framework that stifles research}

Indeed, Europe has to ensure that synthetic biology does not encounter the same problems - a misinformed debate, myths and misconceptions - that coalesced around GM crops and stem-cell research. "Genetically modified crops show that bioengineering discussions are emotive and can become surrounded by misconceptions in the public's mind," Freemont added. "We need to stress the importance of this work and get the ethical and regulatory frameworks in place to reassure the public."

Despite discussions about the details, Europe seems well placed to ride in the vanguard of the synthetic biology revolution. "The USA is only slightly ahead of us-but Europe has the advantage of strong academic and research networks that American researchers rightly envy," Freemont concluded. "The grass isn't always greener on the other side of the Atlantic. Progress may be a bit slow, but the EU is going through the right processes to ensure that Europe maintains or improves our position."

\section{REFERENCES}

Balmer A, Martin P (2008) Synthetic Biology. Social and Ethical Challenges. Nottingham, UK: University of Nottingham

Benenson Y, Gil B, Ben-Dor U, Adar R, Shapiro E (2004) An autonomous molecular computer for logical control of gene expression. Nature 429: 423-429

Gibson DG et al (2008) Complete chemical synthesis, assembly, and cloning of a Mycoplasma genitalium genome. Science 319: 1215-1220

Hale V, Keasling JD, Renninger N, Diagana TT (2007) Microbially derived artemisinin: a biotechnology solution to the global problem of access to affordable antimalarial drugs. Am J Trop Med Hyg 77 (Suppl): 198-202

O'Malley MA, Powell A, Davies JF, Calvert J (2008) Knowledge-making distinctions in synthetic biology. BioEssays 30: 57-65

\section{Mark Greener}

doi:10.1038/embor.2008.168 